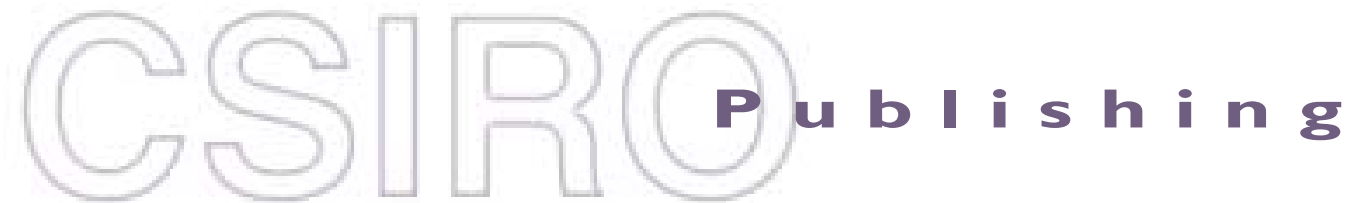

\section{Publications of the Astronomical Society of Australia}

Volume 19, 2002

(C) Astronomical Society of Australia 2002

An international journal of astronomy and astrophysics

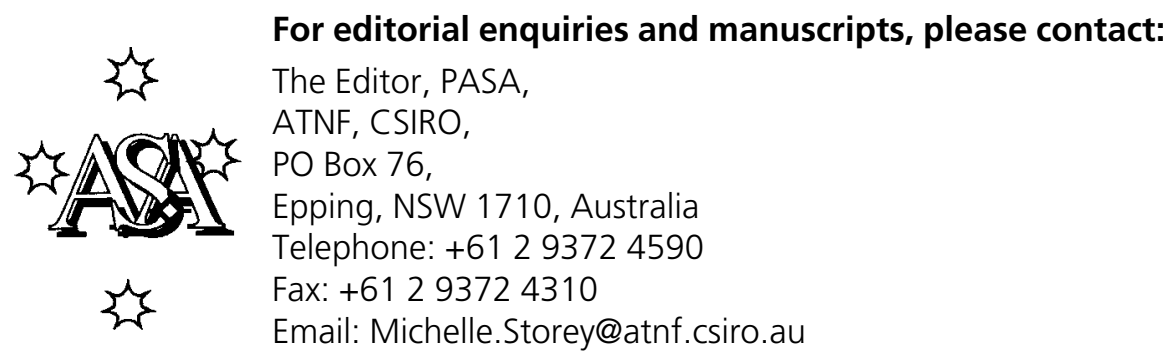

For general enquiries and subscriptions, please contact: CSIRO Publishing PO Box 1139 (150 Oxford St)

Collingwood, Vic. 3066, Australia

Telephone: +6139662 7666

Fax: +61 396627555

Email: publishing.pasa@csiro.au

C S I RO

PUBLISHING Published by CSIRO Publishing

for the Astronomical Society of Australia

www.publish.csiro.au/journals/pasa 


\title{
Probing the pc- and kpc-scale Environment of the Powerful Radio Galaxy Hercules A
}

\author{
Nectaria A. B. Gizani ${ }^{1}$, M. A. Garrett ${ }^{2}$ and J. P. Leahy ${ }^{3}$ \\ ${ }^{1}$ Centro de Astronomia e Astrofísica da Universidade de Lisboa, \\ Observatório Astronómico de Lisboa, Tapada da Ajuda, Lisboa, Portugal, 1349-018 \\ ngizani@oal.ul.pt \\ ${ }^{2}$ Joint Institute for VLBI in Europe, Postbus 2, Dwingeloo, The Netherlands, 7990 AA \\ garrett@jive.nl \\ ${ }^{3}$ Jodrell Bank Observatory, University of Manchester, Nr Macclesfield, UK, Cheshire SK 11 9DL \\ jpl@jb.man.ac.uk
}

Received 2001 July 26, accepted 2001 November 27

\begin{abstract}
We present the kpc-scale behaviour of the powerful extragalactic radio source Hercules A and the behaviour of the intracluster gas in which the radio source is situated. We have found that Hercules A exhibits a strong Laing-Garrington effect. The X-ray observations have revealed an extended X-ray emission elongated along the radio galaxy axis. The estimated temperature of the cluster is $k T=2.45 \mathrm{keV}$ and the central electron density is $n_{\circ} \simeq 7.8 \times 10^{-3} \mathrm{~cm}^{-3}$ which reveals a hot, dense environment in which Hercules A is situated. From the combined study of the radio and X-ray data we have estimated a central value of $3 \lesssim B_{\circ}(\mu \mathrm{G}) \lesssim 9$.

We also present the most recent results from the analysis of the radio data on the pc-scale structure of the radio galaxy, observed at $18 \mathrm{~cm}$ by the EVN-MERLIN array. A faint but compact radio source, coincident with the optical centre of Hercules A, was detected by the EVN at 18 mas resolution. The total flux density of the $\mathrm{EVN}$ core is $14.6 \mathrm{mJy}$. Its angular size is $18 \times 7 \mathrm{mas}$ with a position angle of $\simeq 139^{\circ}$. There is also evidence for extended emission in the NW-SE direction, most probably from the eastern pc-scale jet. If this is true then there is a misalignment between the direction of the pc-eastern and the aligned kpc-scale jets of $\simeq 35^{\circ}$.
\end{abstract}

Keywords: radio continuum: galaxies — galaxies: elliptical and lenticular, cD — galaxies: nuclei magnetic fields — galaxies: clusters: individual (Hercules A, 3C 310) - X-rays: galaxies: clusters

\section{Introduction}

Hercules $\mathrm{A}$ is an extended source at a low redshift of $z=0.154$ with total radio luminosity $\sim 3.8 \times 10^{37} \mathrm{~W}$ between $10 \mathrm{MHz}$ and $100 \mathrm{GHz}$. Its power density at $5 \mathrm{GHz}$ is $\mathrm{P}_{5 \mathrm{GHz}}=6.9 \times 10^{25} \mathrm{~W} \mathrm{~Hz}^{-1} \mathrm{sr}^{-1}$ (assuming $H_{\circ}=$ $65 \mathrm{~km} \mathrm{~s}^{-1} \mathrm{Mpc}^{-1}$ and $q_{\circ}=0$ ). It is classified as intermediate between the two FR classes (Dreher \& Feigelson 1984). With a linear size of $540 \mathrm{kpc}$ and no compact hot spots, Hercules A possesses almost symmetrically extended lobes and two jets which are quite different in appearance (see Figure 1). The western jet shows partial or full ring-like features that form a linear sequence heading from the core to the lobes. The eastern jet has the highest luminosity found so far in a radio galaxy associated with an elliptical galaxy (Dreher \& Feigelson 1984).

Hercules A is identified with a very elongated $\mathrm{cD}$ galaxy (e.g. Sadun \& Hayes 1993; Baum et al. 1996), with absolute magnitude -23.75 at the R band. The HST observations (Baum et al. 1996) have shown that the radio source is associated with the diffuse low surface brightness elliptical galaxy and not with the smaller and brighter companion. The cluster in which the host galaxy is embedded is of a typical Abell richness 0 to 1 (Abell 1958).

\section{The kpc-scale Environment}

Gizani (1997) has made extensive radio total intensity and polarisation multiband, multiconfiguration observations of Hercules A (N. A. B. Gizani \& J. P. Leahy, in preparation; Gizani \& Leahy 1999) using the VLA, and also ROSAT X-ray observations. The observations were at $1665,1435,1365$, and $1295 \mathrm{MHz}$, in the A, B, and C configurations, at 8465 and $8415 \mathrm{MHz}$ in the $\mathrm{B}, \mathrm{C}$, and $\mathrm{D}$ configurations, and we have retrieved and reanalysed the $4873 \mathrm{MHz}$ data (B, C, D configurations). The purpose of these multifrequency observations was to map the Faraday rotation field at high resolution, 1.4 as, combined with the $\mathrm{X}$-ray data on the gas distribution in order to map the magnetic field of the cluster.

The analysis has shown that Hercules A exhibits a strong Laing-Garrington effect (Laing 1988; Garrington et al. 1988), i.e. the western side of the radio emission is more depolarised than the eastern side. The projected magnetic field closely follows the edge of the source, the jets, and the ring structures in the lobes and the field pattern in the two lobes is broadly similar.

Using all seven frequencies of our radio observations, we have found that Hercules A is a steep spectrum radio source with spectral index $\alpha \simeq-1.6$ (we assume that the flux density varies as $\left.S_{v} \propto v^{\alpha}, \alpha<0\right)$. Also the more depolarised western side has a steeper spectrum than the less depolarised eastern side. The core is optically thin, with $\alpha \simeq-1.20$. The jets and the ring-like features have spectral index $\simeq-1.1$, while the surrounding lobes are much steeper, $\simeq-1.6$, which is some evidence for spectral curvature. 


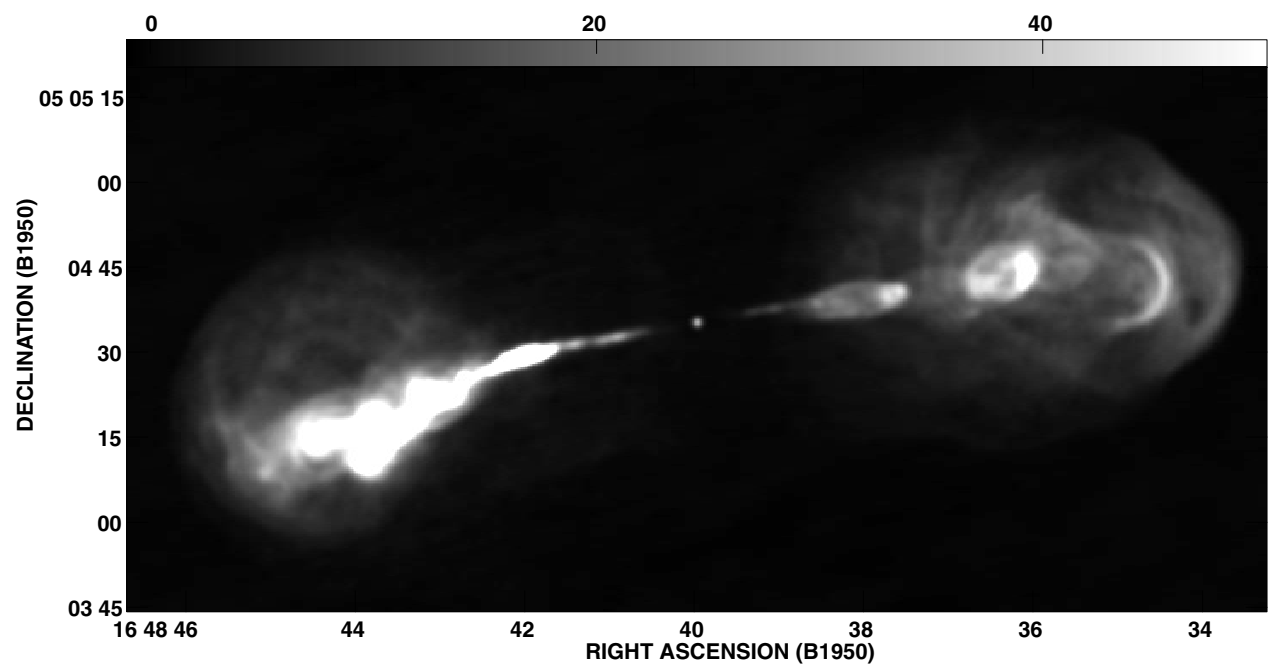

Figure 1 A greyscale map of Hercules A with greyscale flux range -1.0 to $50.0 \mathrm{mJy} / \mathrm{beam}$. The map is at $18 \mathrm{~cm}$, at 1.4 arcsec resolution. The presence of semi rings is apparent mostly in the western lobe. The eastern jet exhibits helical-like features, which could be ring-like but seen in projection.

The X-ray emission of the intracluster gas surrounding Hercules A was observed with the ROSAT PSPC and HRI detectors. These observations have revealed an extended emission elongated along the radio galaxy axis. The estimated central electron density of $n_{0} \approx 7.8 \times 10^{-3} \mathrm{~cm}^{-3}$ reveals a dense environment in which the radio source is situated. Our spectral fitting with hydrogen column density of $N_{H}=6.2 \times 10^{20} \mathrm{~cm}^{-2}$ gives a temperature estimate of $k T=2.45 \mathrm{keV}$. The unabsorbed luminosity of the cluster is $3.16 \times 10^{37} \mathrm{~W}$. The $0.1-2.4 \mathrm{keV}$ luminosity of the core is $2.0 \times 10^{36} \mathrm{~W}$.

From the combined radio and X-ray analysis, by model fitting the Faraday dispersion profile from the radio data and the surface brightness profile from the X-ray data, we have found that the depolarisation is mainly caused by a centrally condensed medium in which Hercules $\mathrm{A}$ is embedded at $\simeq 50^{\circ}$ to the line of sight (Gizani \& Leahy 1999). Most probably this medium is the 'X-ray' hot gas. According to unified schemes the relativistic beaming is causing the depolarisation asymmetry seen between the two sides of the radio emission: the western weak jet and associated lobe is behind the bulk of the gas while the bright eastern jet and lobe are in front. As the radio emission from the lobes travels through this material it is affected by Faraday rotation which causes the depolarisation. Hence the further lobe, on the counter-jet side, is more depolarised.

The external magnetic field is decreasing with radius and Gizani (1997) has estimated a central value of $3 \lesssim$ $B_{\circ}(\mu \mathrm{G}) \lesssim 9$ (see also Gizani \& Leahy 1999).

\section{The pc-scale Environment}

The VLA data provide us with a good understanding of the kpc-scale radio structure of Hercules A. There seems to be a symmetry between the ring-like features of the western jet and the bright helical-like structures of the eastern jet. The features form a sequence which suggests successive ejections from the active nucleus. On the whole the observed wiggles in the large scale jets and the overall symmetry of the envelope of the radio structure may suggest precession or wobbling of the radio axis. The flux of the core is $41 \mathrm{mJy}$ at $18 \mathrm{~cm}$ at $1.4 \mathrm{arcsec}$ resolution. The inner jets of Hercules A together with its optically thin, steep spectrum core, were unresolved with the VLA.

Taking the above into account we have tried to study the pc-scale environment of this powerful radio source using the EVN+MERLIN interferometer at $18 \mathrm{~cm}$. The main goal of the EVN and joint MERLIN observations (Gizani, Garrett, \& Leahy 2000; N. A. B. Gizani, M. Garrett, \& J. P. Leahy, in preparation) was to try to detect any pcscale jet emission on both sides of the central engine, to map the transition region between them and the kpc-scale jets with the highest possible resolution, and to resolve the inner jets in detail. We have observed Hercules A for $11 \mathrm{~h}$ employing phase referencing since the emission from the area of the core was so weak.

There were problems with the MERLIN observations, due to the diffuse, extended structure of this bright source. We have therefore combined our VLA data with the MERLIN data at $18 \mathrm{~cm}$, in order to obtain information of the inner region of the radio source. This work is in progress. Figure 2 shows the EVN detection of the core region.

A compact core is detected with the EVN at 18 mas resolution. Using a model with one Gaussian component we have found the size of the core to be $18 \times 7$ mas at position angle $139^{\circ}$. The error estimate based on rms is $3.6 \times 10^{-4} \mathrm{Jy} /$ beam. The flux density is $\simeq 15 \mathrm{mJy}$, which implies a radio power of $3.6 \times 10^{22} \mathrm{~W} \mathrm{~Hz}^{-1} \mathrm{sr}^{-1}$. The implied brightness temperature of the compact core is $\simeq 2 \times 10^{7} \mathrm{~K}$. The core is still not resolved out at $18 \mathrm{mas}$ and very weak. There is an indication of a real emission from the core region in the NW/SE direction (see Figure 2). If 


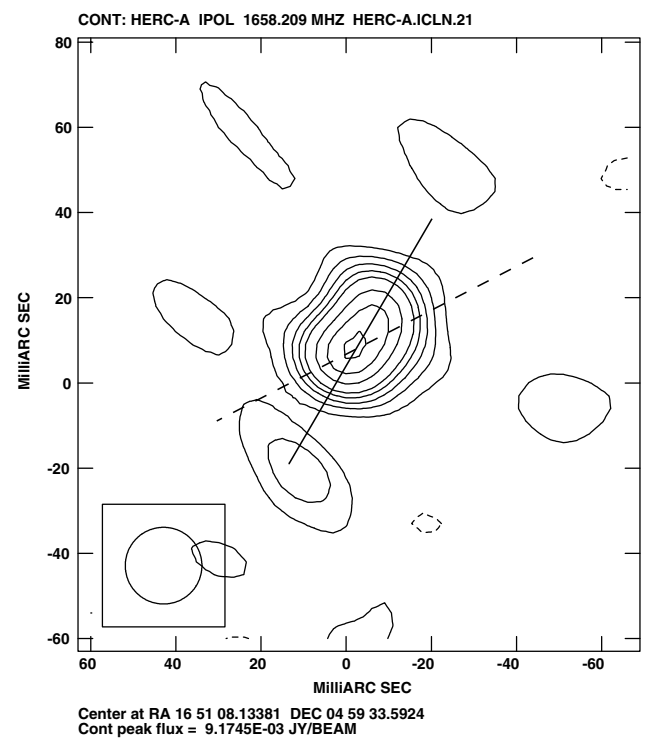

Figure 2 The EVN contour map of the core region of Hercules A. The dashed line indicates the direction of the kpc-scale jets. The solid line shows the direction of the possible NW/SE emission with the current observations (see text). Contours are at levels $-1,1$, up to 10 with increments of 1 , starting at $2.5 \times 10^{-5} \mathrm{Jy} / \mathrm{beam}$.

this is true then it could be interpreted as the emission from the core and one-sided jet: according to Doppler beaming the mas scale jet is always on the same side as the brighter kpc-scale jet (eastern jet in our case). Therefore the core should be located at NW of the observed emission while the SE emission is from the eastern pc-scale jet. If this is indeed the case, then there is a misalignment between the direction of the eastern pc-scale jet, at least, with the larger scale jets of $\sim 35^{\circ}$. This misalignment angle, which is relatively high compared to the ones found in other powerful radio galaxies (Cyg A, Krichbaum et al. 1998; 3C 109, Giovannini et al. 1994, for example), is characteristic of quasars in the CSS class with steep spectrum cores (Fejes et al. 1992, for example, for the OVV quasars 3C 216 and 3C 446 with 'superluminal behaviour', and references therein). This result could be consistent with the suggestion that powerful radio galaxies are the unbeamed counterparts of quasars (Saripalli et al. 1997).

\section{Conclusions and Future Work}

In kpc-scales we have found that Hercules A exhibits a strong Laing-Garrington effect. The depolarisation asymmetry between the two sides can be explained by Hercules A being embedded in a centrally condensed medium at a substantial angle to the line of sight. This medium should be the X-ray emitting gas. The more depolarised western side has a steeper spectrum than the less depolarised eastern side; also the jets and rings in the western lobe have a much less steep spectrum than the lobe material, suggesting that we are witnessing a renewed outburst from the active nucleus. Our X-ray observations of the intracluster gas in the Hercules A cluster have revealed an extended $\mathrm{X}$-ray emission and a possible weak nuclear component. The cluster exhibits a significant concentration of gas towards the centre: it is hot and dense. We estimated the central value of the external magnetic field strength by model fitting the radio and X-ray data. We found that the magnetic field is decreasing with radius and its estimated central value is $3 \lesssim B_{\circ}(\mu \mathrm{G}) \lesssim 9$.

In pc-scales the results came from the EVN detection at $18 \mathrm{~cm}$. About $30 \%$ of the VLA flux from the core area at 18 mas is detected with angular size $18 \times 7$ mas, position angle $\simeq 139^{\circ}$, and error estimate based on rms $3.6 \times$ $10^{-4} \mathrm{Jy} /$ beam. Its brightness temperature is $2 \times 10^{7} \mathrm{~K}$. The core is still not resolved out and very weak. There is evidence for emission extended in the SE direction. This emission is probably from the eastern pc-scale jet, due to Doppler boosting. Then one can conclude that there is a misalignment between the pc- and kpc-scale jet (at least the eastern one) of $35^{\circ}$. Not much is known about the curvature of the pc-, kpc-scale jets for steep spectrum sources versus redshift. This study is within our scientific interest.

For the future, since there is a possibility the counter jet is being obscured by a foreground absorption and a possible toroidal or circumnuclear disk, we are planning optical and infrared observations as well as HI absorption measurements. The latter could prove the presence of a foreground absorption with the galactic column density assumed in our analysis. UV observations, to use together with other wavelength observations, as diagnostic of the ISM around the central region together with the study the UV emission from nuclear region, are also within our plans. High sensitivity VLBI observations at lower frequencies could determine the extent of the eastern pc-scale jet and could search for the presence of a western counterjet. Simultaneous multifrequency VLBI observations could be the method of probing the pc-scale environment of Hercules A.

It could also be interesting to see whether the VLBI core of Hercules A is variable, like Cygnus A (Krichbaum et al. 1998) and 3C 338 (Feretti et al. 1993) for example.

\section{Acknowledgments}

$\mathrm{NG}$ is grateful to the LOC of this very interesting workshop for giving her most of the financial support, without which her participation in the workshop would have been impossible. NG acknowledges the European Commission's TMR 'Access to Large-Scale Facilities' programme, under contract ERBGCHT950012, in order to reduce the EVN+MERLIN data on Hercules A. NG would also like to acknowledge her postdoctoral grant PRAXIS XXI/BPD/18860/98 from the Fundação para a Ciência e a Tecnologia, Portugal, which covered the rest of the expenses for her participation in this workshop, and which supports her work. The European VLBI Network is a joint facility of European and Chinese radio astronomy institutes funded by their national research councils.

\section{References}

Abell, G. O. 1958, ApJS, 3, 211

Baum, S. A., O’Dea, C. P., De Koff, S., Sparks, W., Hayes, J. J. E., Livio, M., \& Golombek, D. 1996, ApJ, 465, L5 
Dreher, J. W., \& Feigelson, E. D. 1984, Nature, 308, 43

Fejes, I., Porcas, R. W., \& Akujor, Chidi. E. 1992, A\&A, 257, 459

Feretti, L., Comoretto, G., Giovannini, G., Venturi, T., \& Wehrle, A. E. 1993, ApJ, 408, 446

Garrington, S. T., Leahy, J. P., Conway, R. G., \& Laing, R. A. 1988, Nature, 331, 147

Giovannini, G., Feretti, L., Venturi, T., Lara, L., Marcaide, J., Rioja, M., Spangler, S. R., \& Wehrle, A. E. 1994, ApJ, 435, 116

Gizani, N. A. B. 1997, Environments of Double Radio Sources Associated with Active Galactic Nuclei, PhD Thesis, Jodrell Bank Observatories, University of Manchester, UK
Gizani, N. A. B., \& Leahy, J. P. 1999, in EVN/JIVE. Symposium No. 4, eds M. A. Garrett, R. M. Campbell, \& L. I. Gurvits (Elsevier Science), NewAR, 43, 639

Gizani, N. A. B., Garrett, M., \& Leahy, J. P. 2000, in Proceedings of the 5th European VLBI Network Symposium, eds J. E. Conway, A. G. Polatidis, R. S. Booth, \& Y. M. Pihlström (Onsala Space Observatory Publications), 19 (ISBN 91-631-0548-9)

Krichbaum, T. P., et al. 1998, A\&A, 329, 873

Laing, R. A. 1988, Nature, 331, 149

Sadun, A. C., \& Hayes, J. J. E. 1993, PASP, 105, 379

Saripalli, L., et al. 1997, A\&A, 328, 78 